Document downloaded from:

http://hdl.handle.net/10251/47635

This paper must be cited as:

Silvestre-Blanes, Javier; Berenguer-Sebastia, Joaquin; Perez-Llorens, Ruben; et ál.. (2012). Garment smoothness appearance evaluation through computer vision. Textile Research Journal. 82(3):299-309. doi:10.1177/0040517511424530.

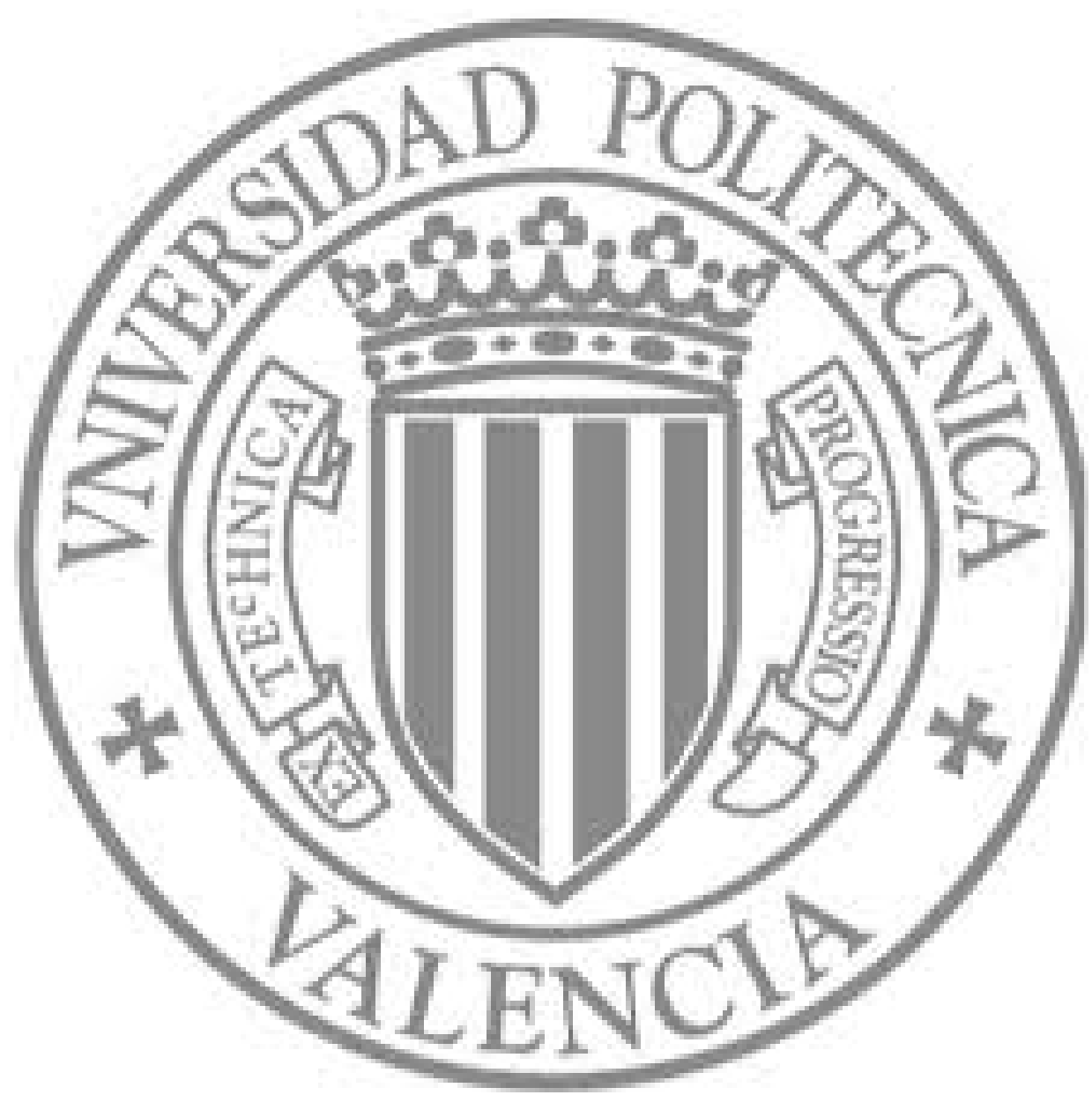

The final publication is available at

http://dx.doi.org/10.1177/0040517511424530

Copyright SAGE Publications (UK and US) 


\section{Garment Smoothness Appearance Evaluation through Computer Vision}

Abstract: The measurement and evaluation of the appearance of wrinkling in textile products after domestic washing and drying is performed currently by the comparison of the fabric with the replicas. This kind of evaluation has certain drawbacks, the most significant of which are its subjectivity and its limitations when used with garments. In this paper, we present an automated wrinkling evaluation system. The system developed can process fabrics as well as any type of garment, independent of size or pattern on the material. The system allows us to label different parts of the garment. Thus, as different garment parts have different influence on human perception, this labeling enables the use of weighting, to improve the correlation with human visual system. The system has been tested with different garments showing good performance and correlation with human perception.

Keywords: Wrinkle evaluation, Image Processing, textile process

\section{Introduction and related work}

Human perception of a garment or of fabric is strongly influenced by how wrinkled it is, as this is one of the most visible properties. Therefore, objective evaluation of this is important in many different situations, such as for example, fabric quality control as it is an important aesthetic parameter; for the design of washing machines and driers or automatic pressing machines, given that the satisfaction of the end user is closely related to the perception of how wrinkled a garment or fabric is. The problems for an objective evaluation are the definition of wrinkled are size of the area on the garment to be evaluated. In this sense, the concept of wrinkled could be interpreted as a special characteristic of the fabric's texture. The definition of this super set can already be considered as ambiguous. Thus, IEEE [1] defines texture as an attribute represented by the spatial distribution of grey levels in the pixels of a particular area. Livens [2] stated that a texture can be defined as a collection of local properties found in the same vicinity of grey levels in a particular area of the image. Another author, Jain [3], stated that a texture is a structure composed of similar patterns and elements, but none of these particularly draw the attention, such that the viewer has an impression of global uniformity. 
Therefore, in order to talk of texture, we have to talk of a window of a determined size, not of a single point of the image which would be a function of the levels of grey in this area. This definition could be valid for fabric, as can be seen in Fig 1 . However, it has no real value for the evaluation of real garments. Therefore, an important limitation that is often overlooked is the difficulty and limitation when using these measurements on real garments which have been printed or have seams, folds, etc. as can be seen in Fig. 2 .

Image processing technologies have been developed for different industrial areas like electronic manufacturing [4], ceramic tiles [5], agriculture [6], paper [7], or in different textile processes [8-12]. Related to the object under study in this paper, in [13] the authors analyze the use of the AATCC standard for the evaluation of seam pucker [14], analyzing the limitations of this type of subjective method, and at the same time demonstrating the need for a wider scale of evaluation. In [15] and [16] the authors analyze the objective evaluation of the level of puckering in garment seams. The garments are placed on a mannequin and the seams are scanned using a 3D laser system. The scanned surface is processed by a 2D filter in order to obtain the pucker profile, eliminating the high frequency components caused by the texture of the fabric, and the low frequency components caused by the three-dimensional contours of the garment. A photometric stereo method to evaluate fabric wrinkle is developed in [17], but is only tested on AATCC standard wrinkle pattern, which has a uniform color. A method for evaluating fabric wrinkles and seam puckers based on fractal geometry over the information obtained with a laser probe is presented in [18]. An important fact to consider in this work is that the AATCC grades do not have equal intervals (as can be seen in Fig. 1) as these replicas (SA: Smoothnest Apperance [19] or WR: Wrinke Recovery [20]) have been constructed subjectively, with no quantitative basis. This fact affects the scales of values provided by the methods that employ these AATCC replicas. Another method based on fractals is presented in [21]. The proposed method was validated using the fractal surfaces produced from the mathematical functions and compared with the box and cube counting methods. The results 
show that the fractal dimension measured by the wavelet-fractal method as well as the surface average mean curvature show the power to clearly discern the grades of wrinkle, smoothness appearance as well as seam pucker, and thus can evaluate the fabric surface roughness objectively and quantitatively. However in both works the method is only used with replicas and the real capacity to measure this parameter in real garments is not evaluated. In [22] the evaluation of roughness is carried out using radon transform and texture analysis, using white samples of $10 \times 12 \mathrm{~cm}$ illuminated at an angle of $30^{\circ}$, which gave rise to a significant limitation in the methods proposed here. In spite of the small dimension of the samples, only their centers were considered. Another work with similar limitations and in which even more elementary measurements were used is [23], where among others, the measurement of intensity is used, a characteristic with many limitations when applied in this field, and which is not often used due to its limited capacity to discriminate between textures. Recently, in [24] the authors presented an interesting 3-D system for fabrics based on stereo vision. However, the size is also limited, and there is not a clear definition between the three figures obtained and the global wrinkle evaluation, or their correspondence with the visual rating.

Therefore, due to the needs of industry and the limitations of available methods, the objective of this paper is to satisfy the need to develop an automatic system for evaluating wrinkling that can:

- Analyze real garments of considerable size.

- Analyze real garments of all colors and print pattern types.

- Analyze the wrinkling on different parts of the garment, and that these have different weightings in function of the importance given by the consumer to each part of the garment. 
Through the work carried out in this paper we have developed an automated system to measure and evaluate wrinkling. This system overcomes the previous limitations of existing systems: range of measurable garments and the subjectivity associated with human evaluation, and which also takes into account different parts of the garment having different weight or incidence on the final evaluation. In next section we present the theoretical framework. In section 3 we give different details of the system developed, including calibration of the evaluation method. In section 4 we present the results obtained, the conclusions and future work.

\section{Theoretical Framework}

This system is based on the projection of laser lines onto the garment, and then reading these lines using the appropriate vision system. In this way, the wrinkles and other similar characteristics of the garment will cause a distortion that can be analyzed through the shape of the laser line detected. The use of a laser line means that it is necessary to move the garment automatically around the capture zone in such a way that, finally, we obtain $N$ straight lines of longitude $L$ (see Fig. 3).

If $\mathrm{f}^{\mathrm{i}}$ is the theoretical straight line and $\mathrm{g}^{i}$ the laser line detected on the garment, the evaluation of the wrinkling on the garment is a function of the error existing between $f^{i}$ and $g^{i}$, denominated $\Theta$, with the final evaluation of the garment given by the average of errors evaluated in the $\mathrm{N}$ lines, using a distance between lines of $\boldsymbol{p}$. However, there are usually distortions present in the garment that are not associated with wrinkling which mean that this error evaluation cannot be measured in the total length $L$ of the straight, but rather has to be done in segments. Thus, if $f_{x 1}{ }^{i, x 2}$ and $g_{x 1}, x^{,}$are the respective functions in the segment $[x 1, x 2]$, we can define this error as: 


$$
\Theta_{x 1}^{i, x 2}=f_{x 1}^{i, x 2}-g_{x 1}^{i, x 2} \equiv f_{x 1}^{i, x 1+1}-g_{x 1}^{i, x 1+1}(1)
$$

where $I$ is the established length of the segment. In this way, the error in the area covered by the line $i$ will be the average of the errors measured in each segment of length $I$, that is:

$$
\Theta_{l, s}^{i}=\frac{s}{L-l} \sum_{j=1, j=j+s}^{L} \Theta_{j}^{i, j+l} ; s \leq l(2)
$$

Where $s$ is the overlap of the segments used to calculate the error obtained in each of the lines. Therefore

$$
\Theta_{l, s}=\frac{1}{N} \sum_{i=1}^{N} \Theta_{l, s}^{i}
$$

Fig. 4 shows the process used to measure the error between the laser straight line obtained (grey lines) and the theoretical straight line (arrows) between the points $x 1$ and $x 1+l$ which would be obtained in the case of a totally smooth surface between these two points. The errors are marked in the darker areas. In this figure, we show a line with two wrinkles to show the effect of the choice of I, always using $s=I$ to allow visualization. In the first case, I=5, was used, which allows detection of an error in the first wrinkle but not in the second. Using $\mathrm{l}=10$ improves detection of the error in the second wrinkle, but reduces the error obtained from the first wrinkle. Using a value of $\mathrm{I}=15$, we obtained a good evaluation of the first wrinkle but no error was detected from the second wrinkle. Using $l=20$, this was the value that gave the highest error, although evidently, this value of I could be useless in other cases.

Fig. 5 shows (separated into two samples to allow visualization) the error obtained using $\mathrm{l}=10$ and $s=5$. Although in the first case, the error obtained is low for both wrinkles, the overlapped segments allow us to improve and obtain the detection of the error more efficiently.

\section{System Developed}

\subsection{Acquisition system}


The system developed can be seen in Fig. 6 . The system uses a belt that is capable of moving one millimeter at a time, at 100 millimeters per second, so that the garment can be scanned with maximum resolution. The belt has a width of $900 \mathrm{~mm}$ so that the system can work with garments in a wide range of sizes, but is also manageable and without excessive costs. With these dimensions, a laser source of $690 \mathrm{~nm}$ generates a line over the garment under analysis (represented in Fig. 6 using a dark line on the shirt), which allows us to obtain information on the smoothness of the garment. This information is captured by three black and white area cameras (labeled as Laser Cameras 1, 2 and 3 in Fig. 6) with a band-pass filter to collect only the laser information from the garment. The number of cameras needed depends on many factors, such as the sensor size, focal length, limitations in the maximum distance between the cameras and the object, number of pixels considered, the resolution needed, etc. In this case we have considered this number of cameras, with $12 \mathrm{~mm}$ focal length optics, and with a sensor of $1 / 2 "$, as the best solution without excessive cost. The cameras are set at a determined angle to collect information from the garment using the laser. Fig. 7a shows on the left the image obtained using a laser source of 15 lines over a garment captured with a conventional color camera and on Fig. 7b we can see the image obtained with the band-pass filter. In spite of the fact that only the central line is in focus, these images allow us to see how this illumination technique lets us adequately collect the information on the smoothness of the garment. On the other hand, as can be seen in these images, the information collected does not allow contrast with the evaluation obtained through experts. In order to be able to do this, we use the upper camera (labeled as color camera in Fig. 6) which allows us to obtain the same image that would be seen by a human observer with the ISO/TR 16323 standard [25], and in this way we can appreciate and evaluate the corrections of the measurements obtained by the system. The use of a laser of $690 \mathrm{~nm}$ means that the system has certain limitations, given that there are fabric colors that absorb this light and which makes it difficult to obtain the straight line. The use of other wavelengths would avoid this problem but would make it necessary to satisfy 
a number of safety requirements that arise when using non-visible laser sources, and this would make development of the research difficult. The results obtained are independent of wavelength, which only affects some colors meaning that in order to overcome these limitations it is only necessary to substitute the laser sources and band-pass filters of the camera with others and add the necessary safety requirements depending on the country.

\subsection{Cameras Calibration Method}

The relative position of each of the four cameras to each other is an extremely important characteristic for the correct functioning of the system, as it allows us to unify the information obtained from the three laser reading cameras and at the same time all this information can be projected onto the color camera image to be labeled and processed later.

For the correct relative calibration of the cameras, we first needed to model each of them, using intrinsic parameters and the distortion vector. The intrinsic parameters of the camera are made up of 4 values $\left(f_{x}, f_{y}, c_{x}, c_{y}\right)$ which define the relationship between the points in reality and their projection on to the camera plane. These are the focal points in the axes $X$ and $Y\left(f_{x}\right.$, $\left.f_{y}\right)$ as well as the displacements in the axes $X$ and $Y\left(c_{x}, c_{y}\right)$.

The distortion was calculated using a method derived from [26], which appropriately models the distortions obtaining a vector composed of 5 elements $\left(k_{1}, k_{2}, k_{3}, p_{1}, p_{2}\right)$, which allows us to correct the distortions on the image introduced by the camera lens: The radial distortions which produces the barrel] effect (an image of a rectangular object is projected as barrelshaped) were modeled using the values $k_{1}, k_{2}, k_{3}$ and the tangential distortions, produced by the misalignment of the lens with the CCD of the camera, were modeled using $p_{1}$ and $p_{2}$.

The calculation of these parameters is carried out using capture of points with a known geometry, in this case a chess board, which allows us to observe us to observe known points from the real world projected onto the camera plane. It is necessary to obtain a sufficiently high number of images of the chess board in different positions in order to calculate the 
parameters of the camera model. Although with only two images it would be enough mathematically, in order to obtain good results from the algorithm due to the error in obtaining the corners and the numerical stability of the algorithms, it is necessary to obtain a greater number of images and also that the position of the chess board is significantly different to collect a good set of points. This process is known as camera calibration, and once it has been carried out no more calculations are necessary unless a camera or optic is changed.

Once the intrinsic and distortion parameters have been obtained, we can coordinate the cameras so that they correspond with each other. This process (see Fig. 8) is similar to the calibration: through the capture of a known object (chessboard) by each pair of cameras, we can obtain a transformation matrix of coordinates $M(3 \times 3)$ which characterize the T movement and $\mathrm{R}$ rotation of one of the cameras. In this way, we know how every pixel from one image has its corresponding pixel in the other image. That is to say, if $p_{\mathrm{c} 1}$ is the projection of one point of the real world over the plane at camera 1 at homogeneous coordinates, and knowing $M$, we can obtain $p_{c 2}$ (where the point will be located as it is seen by camera 2 ) as $p_{c 2}=p_{c 1} M$. With this, for each garment we will obtain a work set of an upper image of the garment in color plus a collection of straight lines and points from the scanning carried out by the laser cameras. Through calibration, we know which point of the upper image the points from the laser correspond to, to be able to look at them adequately according to the user labeling.

\subsection{Laser detection}

Once the correspondence matrices between the cameras of the system are known, along with the location of the laser camera with respect to the upper camera, it is possible to define the search zones for the laser for each camera. The detection process of the laser line is done using the images captured by the laser cameras. The lens of these cameras carries out an IR filter before image capture thus obtaining a greater contrast of the laser line for processing and differentiating the background of the image. The search algorithm carries out vertical 
scans of the image from the laser cameras starting from an initial point of the laser line configured in the set up system. The search zone will be bordered within the limit zones of the adjacent cameras. The laser search is made difficult by (see Fig. 9, which is shown inverted for better visualization):

- The color of the textile, with dark colors, especially blues absorbing more of the light emitted. However, as commented in the previous section, this problem can be overcome by changing the wavelength of the lasers;

- Abrupt wrinkles formed due to the type of garment cause losses in certain parts of the line;

- The appearance of noise.

Due to these factors, the search algorithm should be based on the thickness of the line in order to minimize errors. The pixel that is used to calculate the lines is in the centre in each $Y$ axis of the pixels grouped which belong to the laser. In the case of very pronounced wrinkles, the continuity of the laser line may be lost at some points of the axis $\mathrm{X}$, and so the algorithm must be robust with these cuts, in such a way that the line can be found in all the area covered by the laser cameras.

\subsection{Error Calibration}

Before being able to carry out a qualitative analysis of smoothness based on the system described in section 3.1 it is necessary to evaluate the errors obtained from known patterns and the error produced by these patterns with the method presented here so that we can carry out a calibration $f: R \rightarrow N, \Theta \rightarrow S A$.

To obtain information relative to the SA and model the error $\Theta$, these have been extracted on the replicas that are used to model their textures (see Fig. 1) of the different types of SA with the aim of characterizing $\Theta$ and the laser lines obtained from each SA replica. 
The size of the segment will depend on the resolution of the cameras, on the camera/laser angle, as well as the minimum size wrinkle to be detected. In the case of the SA replicas, it should be possible to evaluate the error along all its length, that is, using I=L. However, due to imperfections in the replicas and the high resolution used, deformations are detected which introduce variations in the final evaluation of the laser line. Fig. 10 shows the variations in the value of $\Theta$ for different values of $I$. As can be seen, although the error should be practically constant and independent of $\mathrm{I}$, significant variations are generated which make it important to make the correct selection of I. Analyzing the AATCC replicas, we have empirically shown that values of I higher of 66 would mean that $\Theta$ collects erroneous information on deformations that are not related to the smoothness of the garment. Finally, the values used for the evaluation were the average $I=\{15,30,60\}$ with $s=15$. To characterize $\Theta$ and $S A$ it is necessary to consider that we are evaluating a texture that is not uniform. Therefore, in the replicas type $S A_{i}$ zones of type $S A_{i+1}$ can be given. Thus, once the value of $\Theta$ for $S A_{5}$ is calculated, to calculate the value of $\Theta$ for $\mathrm{SA}_{4}$, all the segments that can be characterized as type $\mathrm{SA}_{5}$ have been extracted from the replica to obtain the correct average of $\Theta$ for $\mathrm{SA}_{4}$, and so on successively. Fig. 11 shows the evaluation on the WR scale, using the following color codes: $S A_{1}$-red, $S_{2}-$ orange, $\mathrm{SA}_{3^{-}}$yellow, $\mathrm{SA}_{4^{-}}$green, $\mathrm{SA}_{5^{-}}$blue. In this way, we extract the relationship between $\Theta$ and the SAs values that can be see in Fig. 12 .

\subsection{Evaluation method}

The method developed allows us to carry out an SA classification of the smoothness of the garments. As we are dealing with garments and not sections of fabric, these are formed of different parts which can have differing influences on the perception of the user. The program allows us to label the garments once they have been scanned over the image captured by the color camera, so that the zones excluded from the analysis can be indicated (seams, buttons, pleats, etc.) together with the zones that were analyzed in the group to which they belong. 
Thus, having the labeling of the color camera available to us, as well as the correspondence matrices between the color and laser cameras, the wrinkle information provided by laser cameras can be ignored or weighted according to the labeling of its position in the color camera.

Thus, if $w_{j}$ is the weighting of the zone $j$, the equation for the final evaluation of the garment is:

$$
\Theta_{l, s}=\frac{1}{M} \sum_{i=1}^{M} w_{j} \Theta_{l, s}^{i} / \Theta_{l, s}^{i} \in j(4)
$$

Fig. 13 shows an example of a report of a garment. On the left, we can see the image as it was captured by the color camera. On the right, we see how some areas of the garment were selected for analysis, while others were not analyzed (due to seams and buttons in this case). The image on the right shows the SA evaluation carried out using the same color codes. In the lower part of the image, two indices are given in the scale [0-16], index one takes into account only the errors measured, and the weighted index takes into account the weighting of the evaluations according to zone. The information used to reach these values can be seen in the lower tables of the report.

\subsection{Results obtained}

In order to evaluate the system, we carried out tests on the following types of garment: woman's slip, underpants, towels, mini-skirt, swimsuit, child's swimsuit, printed blouse, striped blouse, slip, striped slip, slip with straps, skirt, tracksuit bottoms, trousers, red trousers, pyjama bottoms, baby-romper, bib, sheet, child's jumper, striped jumper, sweatshirt and bedspread. The items were submitted to different types of washing and drying, which gave different results in terms of the final smoothness of the garment. These operations were performed using the norm ISO 6330:2000 [27], using a washing machine type A (front-loading machine) and detergent ECE-A (non phosphate). The drying process using the same norm were type A (line dry), C (flat dry) and E (tumble dry). The final results after each wash and dry 
were evaluated by experts from the Textile Technology Institute (AITEX), scaling exponentially the $\mathrm{SA}_{1}-\mathrm{SA}_{5}$ in a new 1-15 scale. After one operation, the same garments were submitted to the same operation but with a different form of drying. The measurements by the experts were averaged to get the final SA index of the garment. This set was compared with the set of the indexes obtained by the automatic system. In Fig. 14 we can see the Pearson Correlation of these two sets, before the exponential scaling, which was around $90 \%$. These values are considered acceptable, and their improvement is limited by the fact that in the system developed the zones outside of the analysis can be marked, while with the human evaluation system, it is difficult to ensure that these areas are outside of the evaluation. However, there is still room for improvement through better analysis of the different zones.

\section{Conclusions and future work}

The use of methods based on analysis of textures from conventional images imposes significant limitations in the capacity of the system, especially when using real garments. The use of structured light allows us to overcome these limitations of prints and size, making the system viable for evaluation of all types of garments. The next step is change the upper color camera for a stereo vision system, and to perform a wider analysis of human wrinkle perception. The stereo vision system could complement this solution as we would then have in the same machine all the wrinkle information that can be obtained today. With an extended statistical human wrinkle perception, it is possible to characterize adequately how the wrinkles affect human opinion, in terms of wrinkle density and wrinkle depth, so we can map adequately the characteristics captured by the system with human perception.

\section{References}

[1] IEEE Standard 610.4-1990, IEEE Standard Glosary of Image Processing and Pattern Recognition Terminology. New York: IEEE Press, 1990.

[2] Stefan Livens, "Image Analysis for material Characterisation," Universitaire Instelling 
Antwepen. Departament Natuurkunde., 1998.

[3] Rangachar Kasturi, Brian G. Schunk Ramesh Jain, Machine Vision.: MaGraw-Hill, 2005.

[4] Y.S. Ihn et al. "Design of a modified binary region median filtering for micro electronic device assembly manipulations" Inf. Conf. on Control, Automation and Systems, pp. 27492753,2007

[5] Fernando López, José Miguel Valiente, José Manuel Prats, Alberto Ferrer, "Performance evaluation of soft color texture descriptors for surface grading using experimental design and logistic regresion", Pattern Recognition 41, pp. 1744-1755, 2008

[6] Sylvain Villete et al. "Simple imaging system to measure velocity and improve the quality of fertilizer spreading in agriculture", J. Electron. Imaging, pp. 031109/1-031109/10, 2008

[7] Ferrante neri, Ville Tirronen, "Memetic differential evolution frameworks in filter design for defect detection in paper production", Evolutionary Image Analysis and Signal Processing, SCO 213, pp. 113-131, 2009.

[8] Rocco Furferi, Lapo Governi Monica Carfagni, "A real-time machine-vision system for monitoring the textile raising process," Computers in Industry 56, pp. 831-842, 2005.

[9] Y.S. Wong, G.S. Hong W. Wanf, "Flank wear measurement by succesive image analysis," Computers in Industry 56, pp. 816-830, 2005.

[10] A. Kumar, "Computer-Vision Vision-Based Fabric Inspection System," IEEE Trans. on Ind. Elec. 52(4), pp. 1073-1079, 2005.

[11] Javier Silvestre, Javier Muñoz Rubén Pérez, "Defect Detection in Repetitive Fabric Patterns," in Int. Conf. Visualization, Imaging and Image Processing, Benalmadena, 2004.

[12] Rubén Pérez, Javier Muñoz Javier Silvestre, "System integration of a mixed fibre detection process in yarn packages by computer vision," in ETFA, Catania, 2005, p. Int. Conf. Emerging Technologies for Factory Automation.

[13] M. Mori, M. Niwa S. Kawata, "An experiment on human sensory measurement and its objective measurement: Case of measurement of seam pucker level," Int. Journal of Clothing Science and Technology 9(3), pp. 203-206, 1997.

[14] AATCC, "Test Method 888-1992. AATCC Technical Manual," 1993.

[15] D. Lu, J.M.K. Macalpine, C.L.P. Hui J. Fan, "Objective Evaluation of Pucker in ThreeDimensional Garment Seams," Textile Research Journal 69(7), pp. 467-472, 1999.

[16] F. Lui J. Fan, "Objective Evaluation of Garment Seams Using 3D Laser Scanning Technology," Textile Reseach Journal 70(11), pp. 1025-1030, 2000. 
[17] X. B. Huang X. B. Yang, "Evaluating Fabric Wrinkle Degree with a Photometric Stereo Method," Textile Reseach Journal 73(5), pp. 451-454, 2003.

[18] J.y. Lee T. J. Kang, "Objective Evaluation of Fabric Wrinkles and Seam Puckers Using Fractal Geometry," Textile Reseach Journal 70(6), pp. 468-475, 2000.

[19] AATCC Committee RA61, "AATCC Test Method 124-2010, Smoothness Appearance of Fabrics after Repeated Home Laundering," 1967.

[20] AATCC Committee RR6, "AATCC Test Method 128-2009, Wrinkle Recovery of Fabrics: Appearance Method," 1968.

[21] S.C. Kim, I.H. Sul, J.R. Youn, k. Chubg T.J. Kang, "Fabric Surface Roughness Evaluation Using Wavelet-Fractal Method. Part I: Wrinkle, Smoothness and Seam Pucker," Textile Reseach Journal 75(11), pp. 751-760, 2005.

[22] S.A. Hosseini Ravandi, M. Youssefi M. Mohri, "Objective evaluation of wrinkled fabric using radon transform," Journal of the Textile Institute 96(6) , pp. 365-379, 2005.

[23] S. Msahli, B. El abed, F. Sakli R. Zaouali, "Objective evaluation of multidirectional fabric wrinkling using image analysis," Journal of the Textile Institute 98(5), pp. 443-451, 2007.

[24] M. Yao, B. Xu W. Yu, "3D Surface Reconstruction and Evaluation of Wrinkled Fabrics by Stereo Vision," Textile Research Journal, vol. 79, no. 1, pp. 36-46, 2010.

[25] ISO/TR 16323-2003, Textiles -- Three dimensional measuring apparatus for fabric appearance.

[26] D. C. Brown, "Close-range camera calibration," Photogrammetric Engineering, vol. 37, pp. 855-866, 1971.

[27] ISO 6330:2000 Textiles - Domestic washing and drying procedures for textile testing 


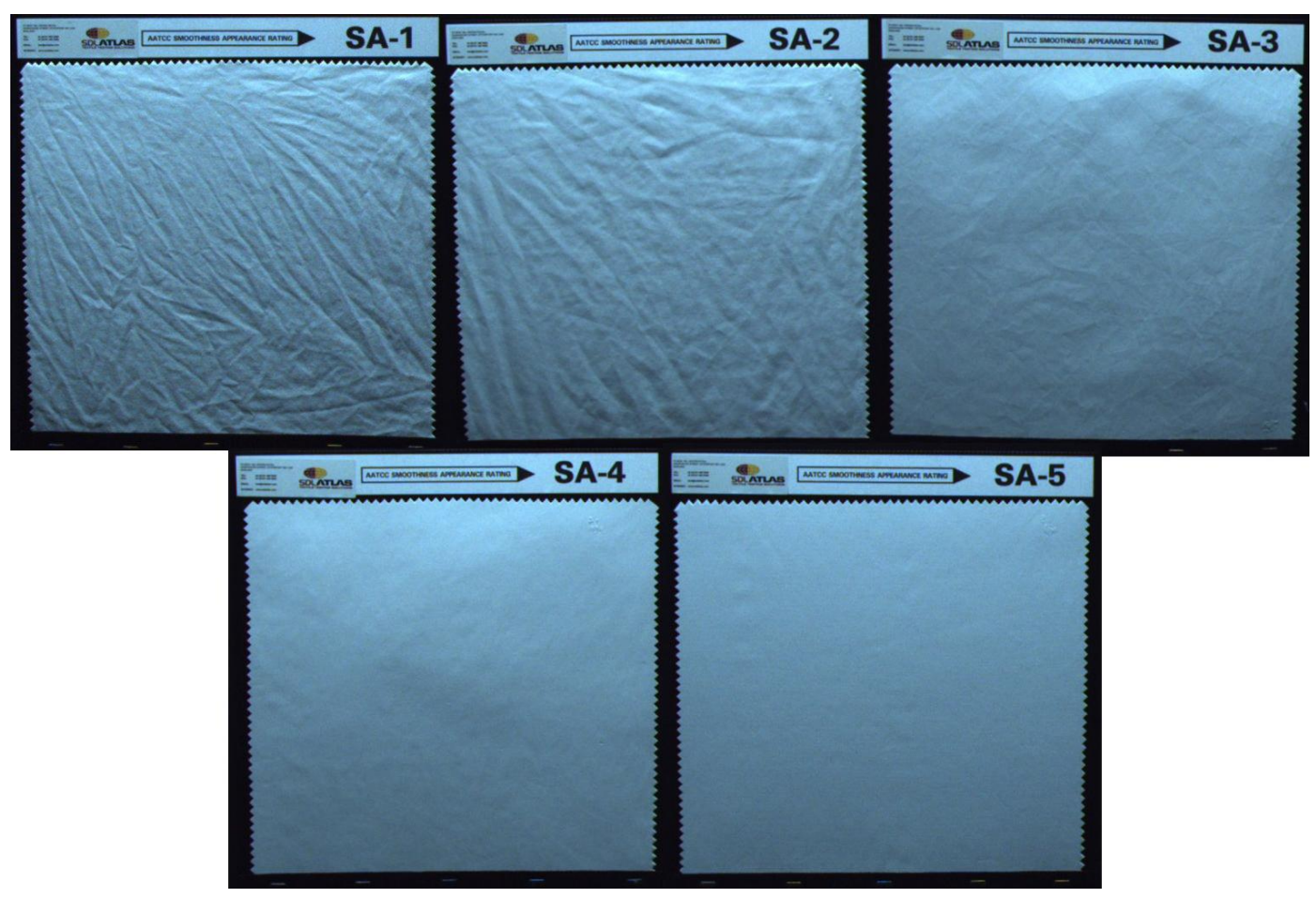

Fig. 1 AATCC Smoothness Appearance Rating

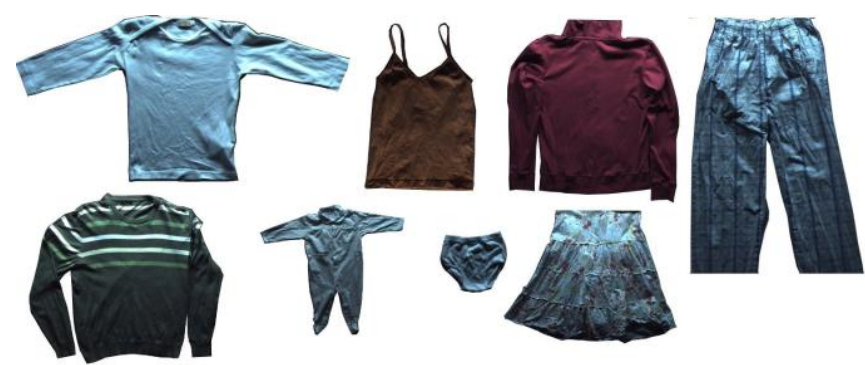

Fig. 2 Real garments 


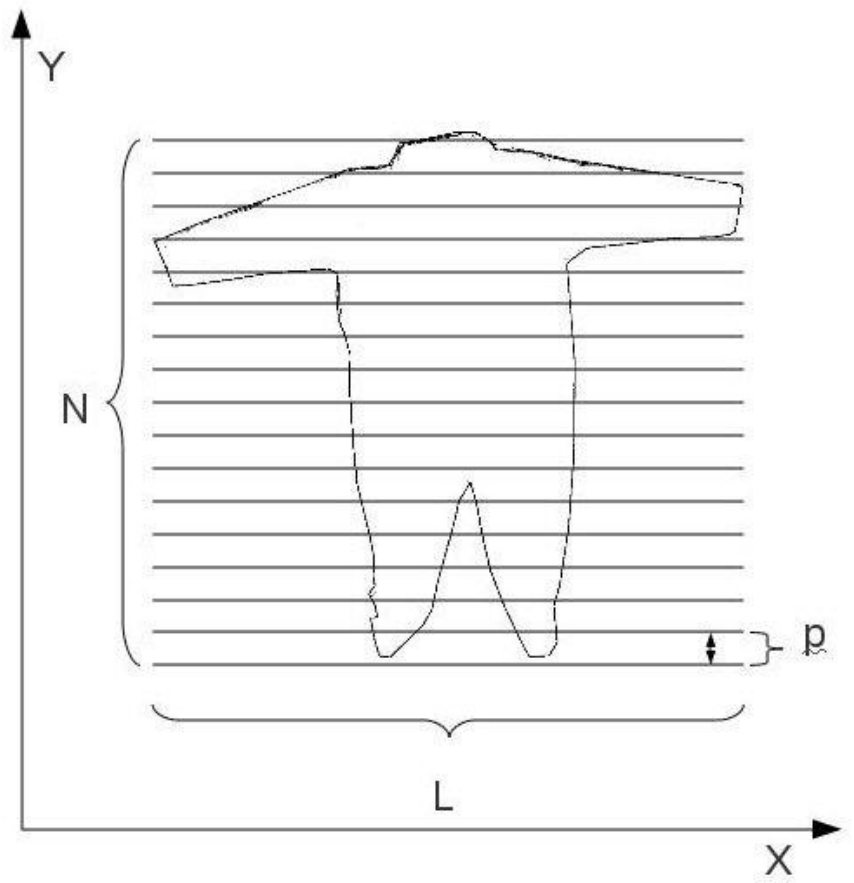

Fig. 3 Laser Lines over a garment

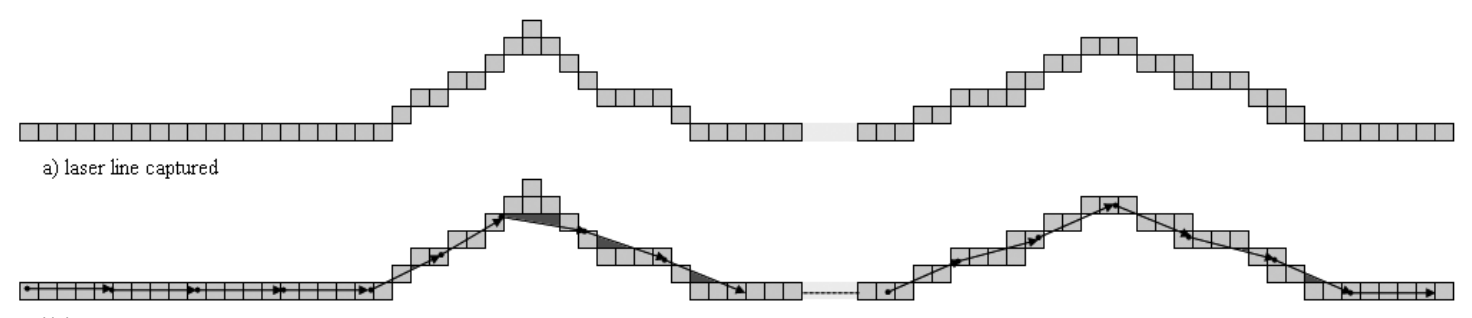
b) $1=5$

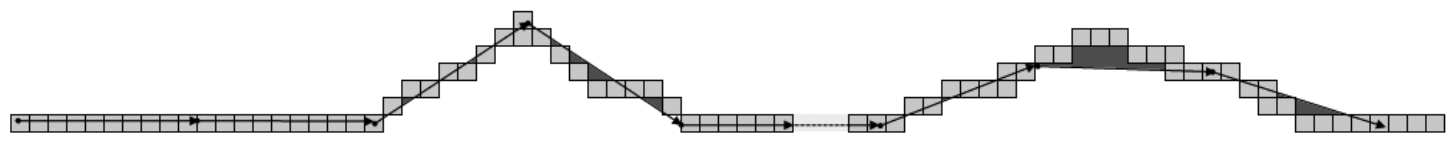
c) $1=10$
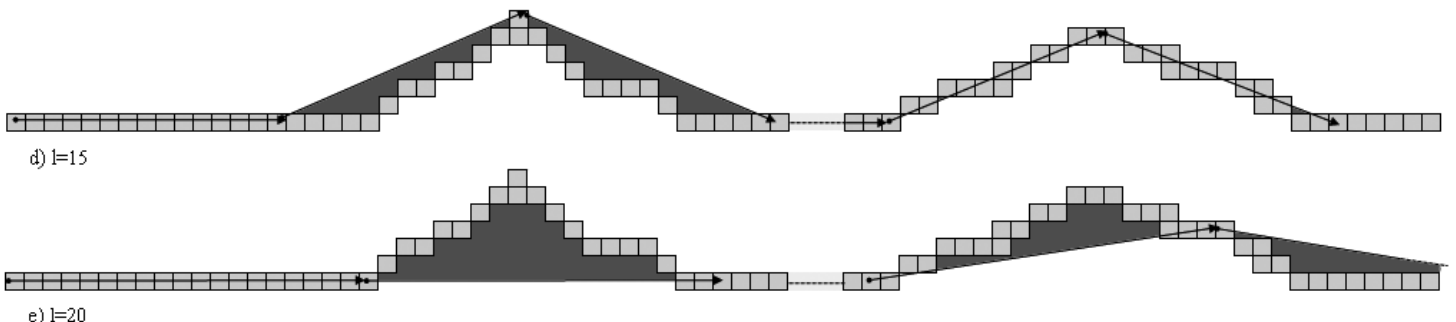

Fig. 4 Example of Error Evaluation for different I values 


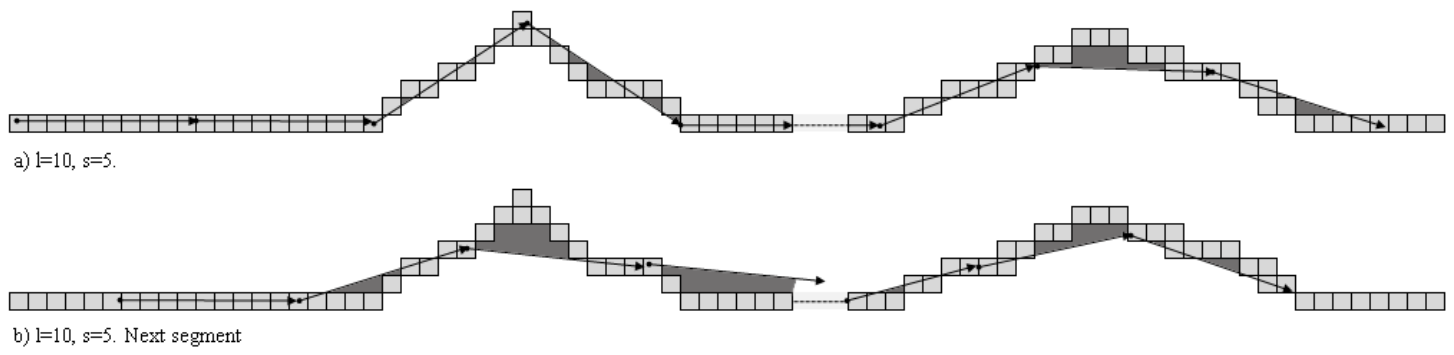

Fig. 5 Example of Error Evaluation with $s<\mathrm{l} / 2$ 


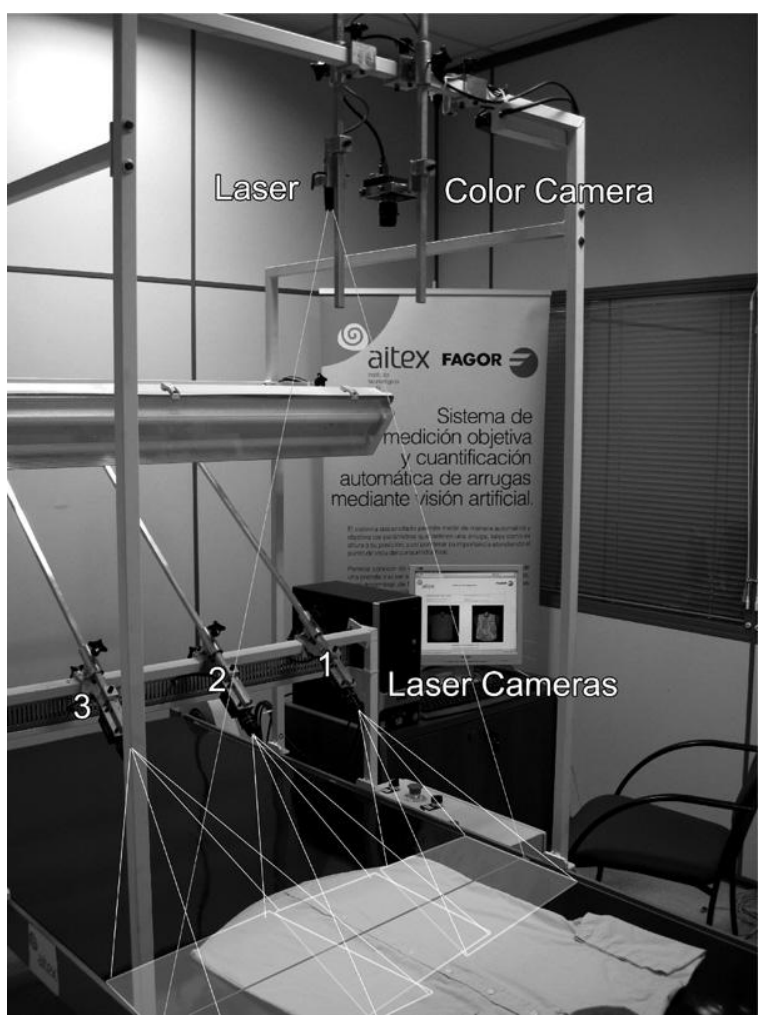

Fig. 6 Wrinkle evaluation acquisition system

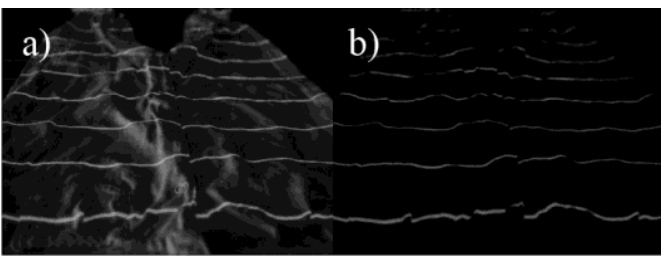

Fig. 7 An example of structured light on a garment

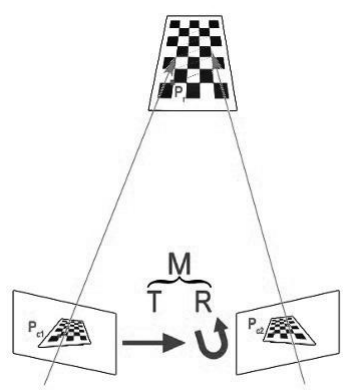

Fig. 8 Calibration Process 


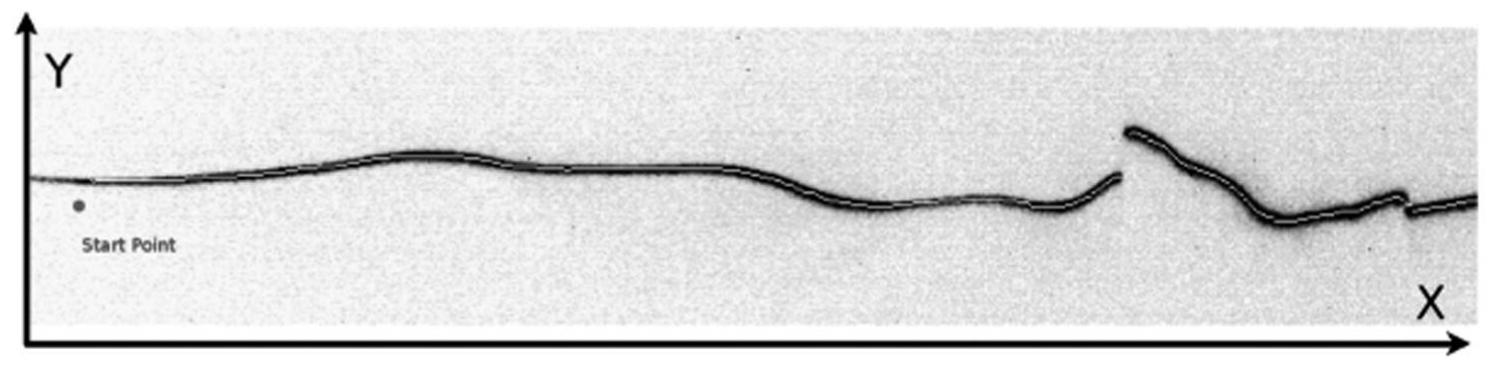

Fig. 9 Laser Search

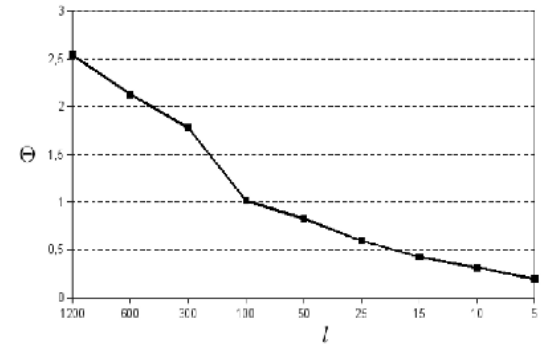

Fig. $10 \Theta$ for different I values

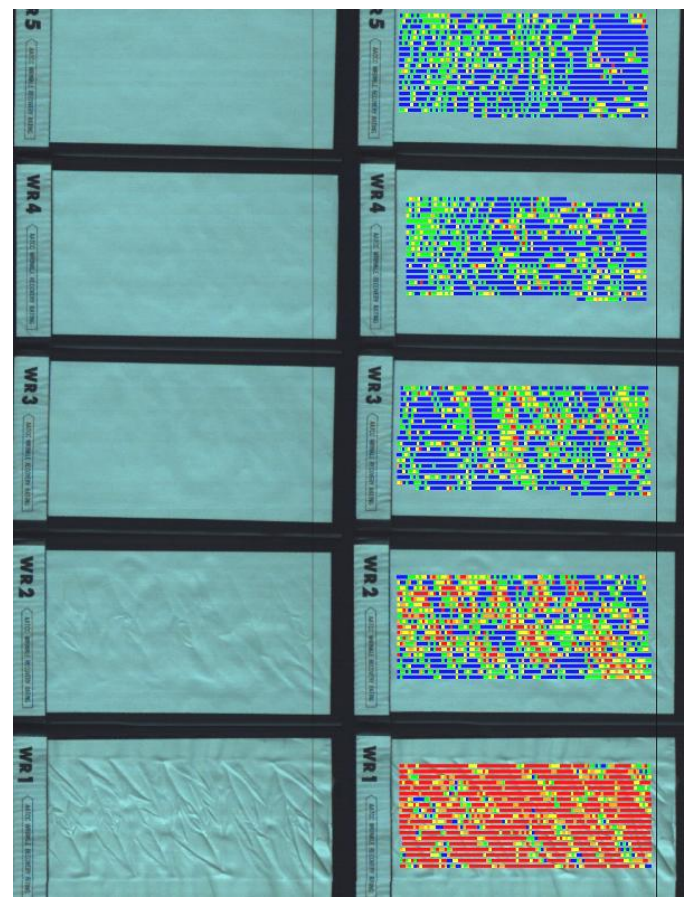

Fig. 11 Evaluation 
Relation Error / AATCC SA

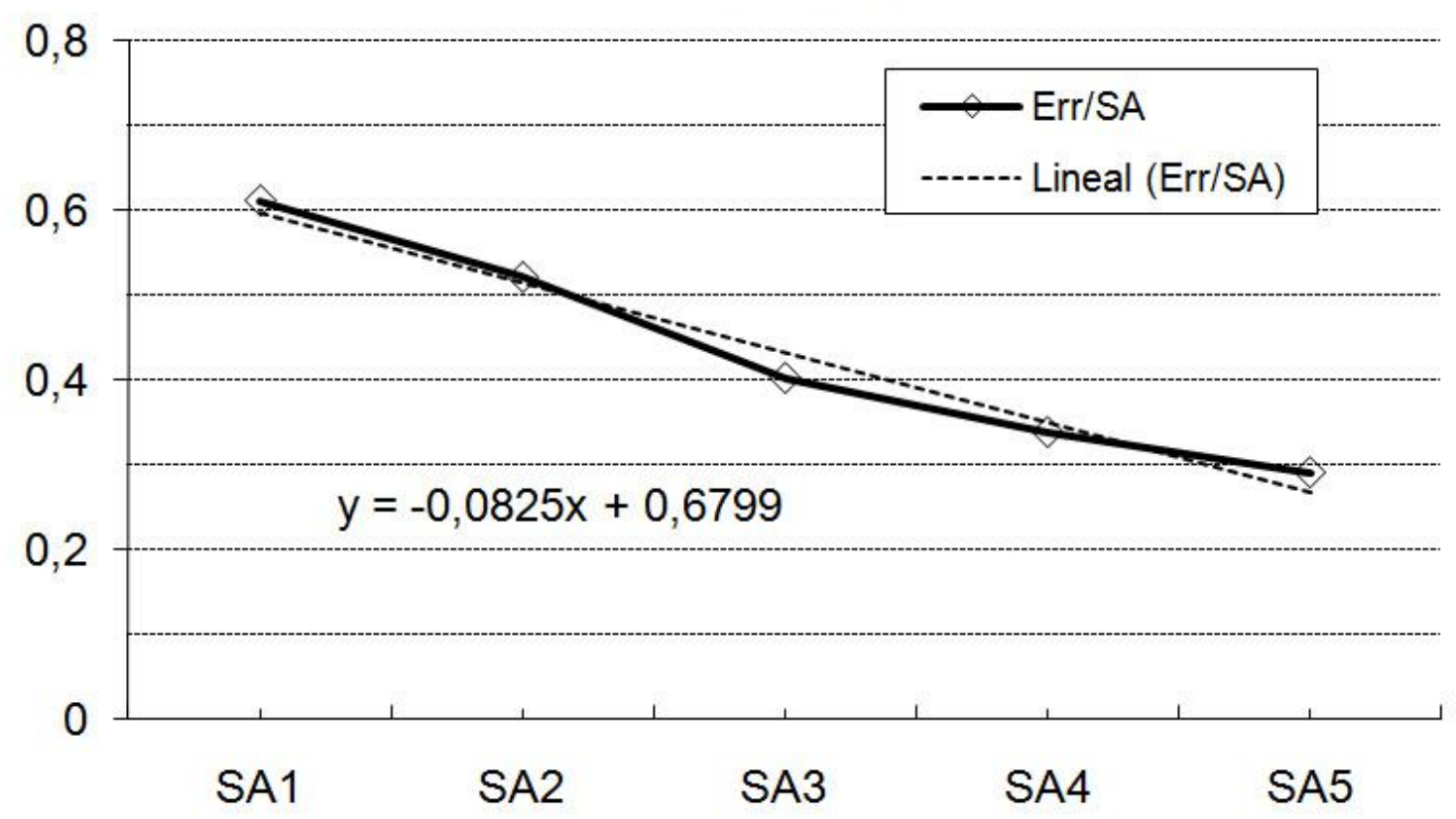

Fig. 12 Relation SA/ $\Theta$ 


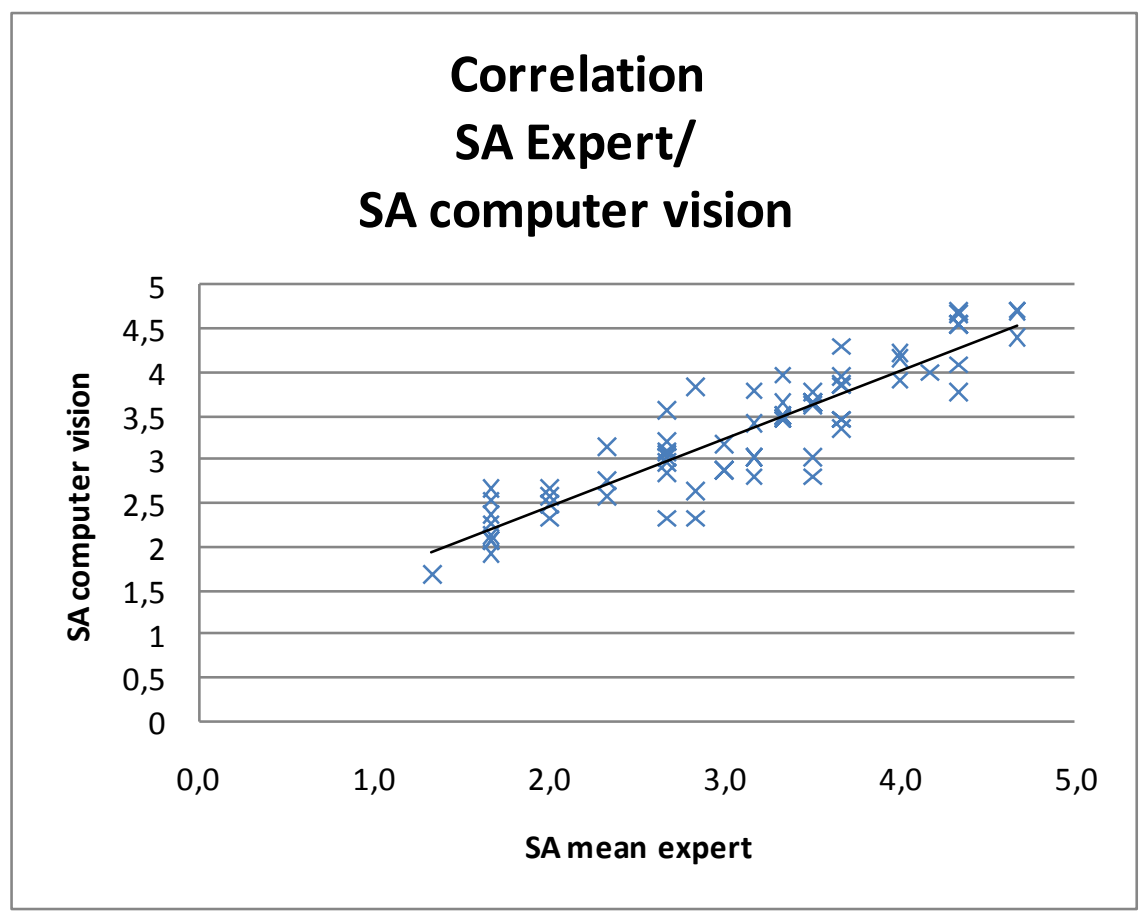

Fig. 13 Pearson Correlation 


\section{aitex}

\section{Smoothness Report}

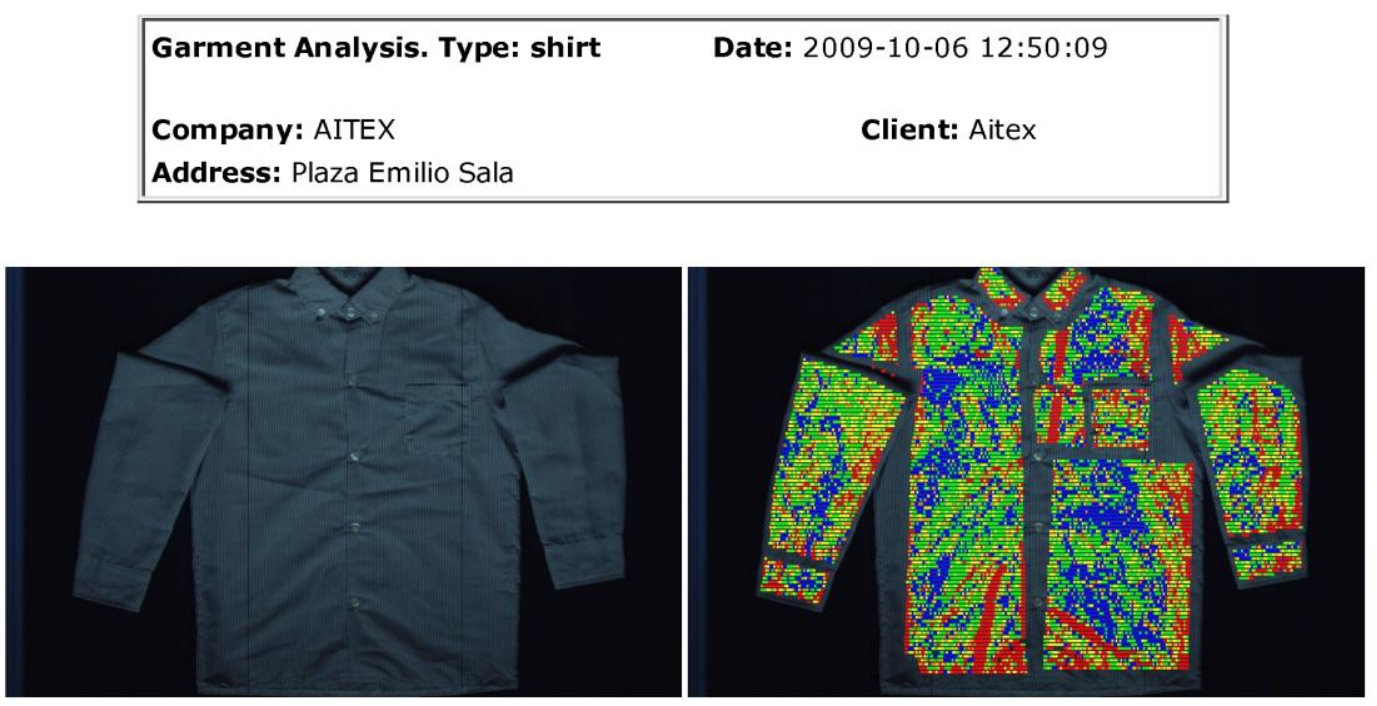

Index: $\mathbf{3 . 3 4}$

Weighted Index: $\mathbf{3 . 0 0}$

\begin{tabular}{|c|c|c|c|c|c|c|c|}
\hline & SA-1 & \multicolumn{1}{|c|}{ SA-2 } & \multicolumn{2}{|c|}{ SA-3 } & \multicolumn{1}{|c|}{ SA-4 } & & Number of samples \\
\cline { 2 - 5 } Windows: & 2745 & 1463 & 3377 & 5133 & 3655 & & 16373 \\
\hline Percentage: & $16.77 \%$ & $8.94 \%$ & $20.63 \%$ & $31.35 \%$ & $22.32 \%$ & & Index \\
\hline
\end{tabular}

Report by zones:

\begin{tabular}{|c|c|c|c|c|c|c|c|c|c|c|c|c|c|}
\hline & \multicolumn{2}{|c}{ SA-1 } & \multicolumn{2}{c|}{ SA-2 } & \multicolumn{2}{c|}{ SA-3 } & \multicolumn{2}{c|}{ SA-4 } & \multirow{2}{*}{ SA-5 } & \multirow{2}{*}{ Index } & W. \\
& Indez & Weight \\
\cline { 2 - 12 } & Windows & $\%$ & Windows & $\%$ & Windows & $\%$ & Windows & $\%$ & Windows & $\%$ & & & \\
\hline Sleeve & 769 & $17.42 \%$ & 465 & $10.53 \%$ & 1140 & $25.83 \%$ & 1421 & $32.19 \%$ & 619 & $14.02 \%$ & 3.15 & 0.58 & $18.29 \%$ \\
\hline Cuffs & 117 & $23.17 \%$ & 48 & $9.50 \%$ & 104 & $20.59 \%$ & 139 & $27.52 \%$ & 97 & $19.21 \%$ & 3.10 & 0.45 & $14.63 \%$ \\
\hline Pockets & 109 & $24.89 \%$ & 66 & $15.07 \%$ & 95 & $21.69 \%$ & 103 & $23.52 \%$ & 65 & $14.84 \%$ & 2.88 & 0.70 & $24.39 \%$ \\
\hline Neck & 97 & $38.65 \%$ & 33 & $13.15 \%$ & 60 & $23.90 \%$ & 54 & $21.51 \%$ & 7 & $2.79 \%$ & 2.37 & 0.46 & $19.51 \%$ \\
\hline Front & 1653 & $15.36 \%$ & 851 & $7.91 \%$ & 1978 & $18.37 \%$ & 3416 & $31.73 \%$ & 2867 & $26.63 \%$ & 3.46 & 0.80 & $23.17 \%$ \\
\hline
\end{tabular}

Fig. 14 Example of a Smoothness report 